\title{
Design and Analysis of Reconfigurable DMTL phase Shifter for High Speed Applications by Mechanical Tuning
}

\author{
G. Srihari, T. Shanmuganantham
}

\begin{abstract}
Tremendous advancement in the field of Radio-frequency were developed through Micro-fabrication techniques, these technologies miniaturize device in a micro-scale behavior for improved device performances. These technologies are developing rapidly due to its distinct features and wide usage in various applications ranging from switches to sensing devices. The principal behind this work is to build a MEMS based Reconfigurable DMTL phase shifter based on RF MEMS switch with improved device phenomenon like switching speed, low actuating voltage, losses and stress factors. The RF switch is build in a series behavior through co-planar waveguides. Generally switching speed is a major concern in RF MEMS devices because of the presence of the actuating elements within the structure which in turn hinders the device ability to function. The phase shifter designed helps in improving the overall switching speed of the device without electrical or dimension alternation of the device. In this paper, we are implementing a PUSH PULL based RF MEMS reconfigurable switch which was structurally altered based on triangular cantilevers in order to enhance the device switching speed. This would develop and enable better application in terms of reconfigurable phase shifter which can be operated at the wider bandgap applications. Even the resonant frequency enhancement was made which provokes an increase of $13 \%$ in terms of the switching speed.
\end{abstract}

Keywords : DMTL, RF MEMS Switch, Switching speed, Mechanical Tuning, Reconfigurable

\section{INTRODUCTION}

In the growing technological standards, the Micro-electro-mechanical system (MEMS) had grown to a larger extent making itself feasible for wide range of applications ranging from switches to that of sensing device. These include the accelerometers, sensor gradient devices and other switching devices like RF, trigger based etc. Mainly RF MEMS switch had gained significant response because of its wide usage in the industrial applications. These technologies are more advantage in terms of their sizing, cost factors and

Revised Manuscript Received on March 11, 2020.

* Correspondence Author

T Srihari*, his/her Department of Electronics Engineering School of Engineering \& Technology, Pondicherry Central University, Pondicherry., India., Email: srihari.nan@gmail.com

T.Shanmuganantham, Department of Electronics Engineering School of Engineering \& Technology, Pondicherry Central University, Pondicherry., India.

(C) The Authors. Published by Blue Eyes Intelligence Engineering and Sciences Publication (BEIESP). This is an open access article under the CC BY-NC-ND license (http://creativecommons.org/licenses/by-nc-nd/4.0/) power consumptions without any deviations in the performance scale[1-2]. The main biggest significant factors is developing the RF MEMS switch through micro-fabrication which relies on the silicon based material which tends to be a general material in terms of IC fabrication. This silicon materials based micro-structures tend to inherit better mechanical properties which helps in building stable devices. The RF MEMS switch is defined to be as an integrated device which utilises a micro mechanical based movement to attain a potential in terms of the RF transmission line. Recently RF MEMS switch is commonly used as block in various communication systems due to its simpler architecture and primary tendency in handling the signals. In the communication system, the MEMS switch has a primary importance as a critical element like phase array for using the application in terms of lower power systems. Compared to the conventional switches, the advanced RF MEMS switch tends to establish high linear profile, less power, minimum losses. Especially when compared to switches based On FET diode or P-I-N structures[3]. The Beam based RF MEMS switch are generally fixed beam based cantilever. In fixed beam based switches the cantilever are fixed at one direction and are movable at other one. The electrodes are designed in such a way that when potential is induced, this intern generated the electrostatic repulses which then causes the beam micro structures to have controllable mechanical motions. This is referred as mechanical deflection, which in turn causes the circuit to open or close for enabling the switching actions. The RF MEMS switch been designed for the micro structure application generally holds a substrate comprising of electrodes, anchors and some contact materials. Chen et al introduced a contact angle based switches which holds a dimple lines in order to reduce the sensitivity of the cantilever tip. Kaneria et al later developed cantilever based switch which performs a static analysis. Then khodaday et al modelled a device based on RF serial MEMS switch with lower actuating potential. The developed potential is about $4.2 \mathrm{~V}$ and with that of return loss upto $26 \mathrm{~dB}$. The RF passive components were introduced based on the MEMS technology[3]. When compared to other RF devices, phase shifters play a significant role in the communication phenomenon. Phase shifter is defined as a double port network which tend to provide differences in phase between the input and the throughput wave, which is in turn controlled through the signal. The phase shifters are implemented for various application ranging 
from phase array antennas to wide band applications, hence they should be of less weight, low loss factors and of minimal cost. These can be overcome through RF MEMS design. Generally these phase shifters are classified into categories like shifter based on switch line and other one is the distributed MEMS transmission line shifter (DMTL).

DMTL are built using RF switches. These switches have high potential in applications like Sensor technology and Wireless communications. These switches are accelerated based on piezoelectric, electromagnetic forces, and electrostatic actuation system are the principle technique for its simpler structures and can be effectively reconfigured. But during the implementation of switches in applications their occurs complexity in tuning the switching speed of the switches. In order to achieve the switching speed, spring constant value needs to be reduced and this can be performed through minimizing the device size and its material manipulation. But this reducing also results in lower responsive time. In this paper we tend to work on enhancing the switching speed of the RF MEMS switch which was integrated with the DMTL phase shifter, where the shifter design was altered using triangular cantilever structures.

\section{BACKGROUND OF RF MEMS SWITCH AND THE PROPOSED DESIGN MODEL}

The RF MEMS switch been integrated with DMTL phase shifter is actuated through electrostatic forces which is in turn explained based on the concept of parallel plate capacitors, where one of the end is moving and the another is fixed. The moving plate is actuated though the mechanical cantilever structures or a beam kind at micro level and the lower plan acts as the transmission waveguide line. The device where wave tendency is very low, the device is termed to be the DMTL shifter. In this shifter, capacitance variation was altered as the actuating element causes deflection with the push and pull device functions. The deflection created in the mechanical actuators are controlled through the voltage been generated thus altering the capacitance values through which the necessary phase differences are been achieved. This phenomenon was defined as the different state characteristics of the MEMS switch, here the switches are correlated with the bridges. In order to achieve the necessary phase difference, the switches are connected in series. Thus RF switches plays a key role while designing the DMTL phase shifter. Enhancing and improving the design of RF switches can improve the performance of the DMTL phase shifter. Initially the DMTL shifter was first designed in 1999 holding a frequency range of about 18-40Ghz[4-6]. Then later the design was improved through using uni-pole multi switches, digital phenomenon of the switches were introduced. Among all the DMTL shifter been reported, the silicon based shifter holding silicon dioxide as a material for dielectric tend to have minimum loss concentration at the larger frequency ranges. Then with the introduction of the micro-machining technology, the constraints in relation to the material usage and implementation were neglected and materials like copper and metal electrodes were implemented. Since the mechanical actuating principles are applied, the mechanical structures are subjected to analysis based on the stress and strain factors and this is performed through addition of load to the structures. While choosing the material for designing the micro structure, certain properties of the materials need to be evaluated like its elasticity and also the young modulus of the particular materials these both acts as a principle factors which tend to decide the peak performance and efficiency of the device based on stability with respective to the working hours. In many mechanical structures, stability factors are eliminated and RF MEMS switch are tuned through potential been generated and not through the mechanical phenomenon. The switch speeds are evaluated through the charging and discharging cycles of the devices. The proposed RF MEMS switch are build using a triangular configuration. These triangular shaped structure acts as the transmission waveguide which is interfaced between the input and the throughput systems, this in turn enhances the charge flow and also improves the parameters like the switching speed and resonant frequency ranges. In this configuration, the weight of the switch is very minimal which helps in achieving better switching speed. In this stress factor are induced through creating a hole in the centre of the mechanical structures which evens up the stress factor and also helps in reducing the potential voltage needed to actuate the switch.

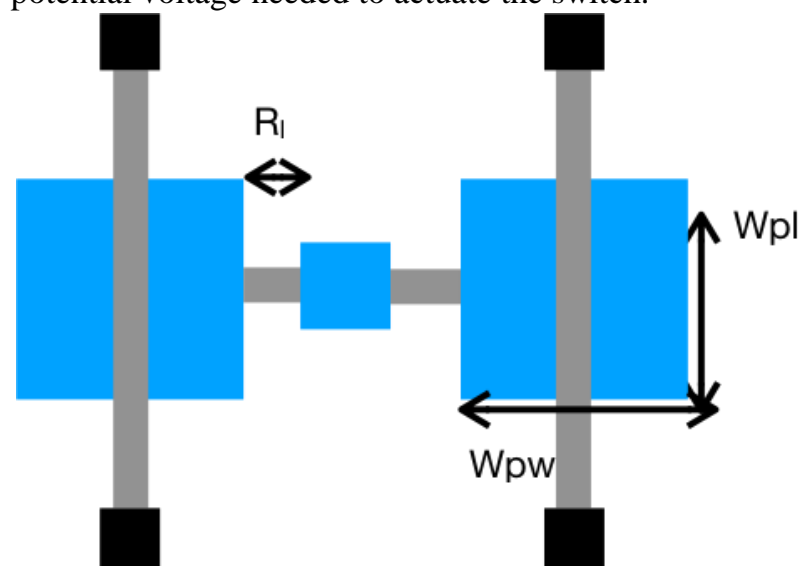

Fig 1 Reconfigurable RF MEMS switch[20]

\section{A. DMTL phase shifter Design}

The DMTL phase shifters were build using the RF MEMS switch and they were integrated across the transmission waveguides which is interrelated between the input and output system and this particular system is considered as alternative conventional system for that of the switches based on the solid state devices[7]. RF MEMS switch plays a significant role in making an efficient DMTL shifter for this they require parametric attributes based on performance, switching speed and efficient and parallely they face challenges in terms of fabrication of the design and capacitance building. In many devices, the mechanical structure been used is cantilever or like beam structure, where the major challenge is associated with stability of the structures which in turn changes the device performance and also causes variation in terms of material change or frequency variations. 
Thus mechanical structures have to be designed which are stable and whose switching speed are improved. The phase difference is based on the incoming wave been generated which in turn depend upon the wavelength factors along with impedance phenomenon. This can be achieved through the switch series configurations on the transmission waveguides. In few DMTL phase shifter design, the capacitance and the inductance values are been considered for improving the device efficiency and performance scale.

\section{RESUlts AND Discussion}

\section{A. Geometry Consideration of RF MEMS Switch}

The RF MEMS switch been designed based on triangular mechanical structural configurations for a higher frequency value ranging from 18 to $40 \mathrm{GHz}$. The beam is developed of $50 \Omega C P W$ which is in turn fabricated onto a silicon wafer acting as a base substrate. The silicon wafer dielectric constant value is given as $\varepsilon r=11.9$. The beam is designed using materials like copper, aluminium, gold and copper which is in parallel with the transmission waveguide. Silicon Dioxide layer is introduced between the beam and the transmission line holding a dielectric constant of about $\mathrm{\varepsilon r}=$ 7.6, of thickness $0.1 \mu \mathrm{m}$ [8-9].

$$
V_{p i}=\sqrt{\frac{0.1708 g_{0}^{3}}{\varepsilon_{0} w_{m o} l_{m e}^{3}}\left(\frac{K_{e f f}}{m}\right)-\frac{0.0279 A}{g_{0} \varepsilon_{0} m}-\frac{27.978 \hbar c}{\varepsilon_{0} g_{0}^{2} m}}
$$

The triangular mechanical structure efficiency holding RF MEMS switch are include based on the parameters like switching speed, resonant frequency and the stress factors been induced. The design of RF MEMS switch are initialised thought the selection of actuating phenomenons. The primary aim of the study is to build a triangular cantilever configuration based on RF series switch with reduced potential during actuation. The equation which governs the voltage phenomenon of the RF MEMS switch are given as

In this $\mathrm{K}$ represents the spring constant of the cantilever beam architecture, Permittivity is indicated as $\varepsilon 0$, height of the beam as g0 and finally the area of the structure is given $\mathrm{W}$. From the equation it is observed that potential been generated is directly proportional to $\mathrm{K}$ and $\mathrm{H}$ value of the beam and also the voltage generated during the actuation phenomenon is also inversely proportional to the relative permittivity and surface area of the configurations. Thus in order to reduce the voltage value, the $K$ and $H$ value need to be decrease or increase of the surface area. But reducing the height of the beam might result in isolation factors and also will impact other parameters. For a better design and performance, cantilever beam length increase should be minimal. The surface of the electrode indicated as $\mathrm{W}$ depicts the effective area of the beam structure and they can be increase through improving the pull down beam electrode configuration.

But in this paper we have concentrated on the speed of the DMTL, through improving the switching speed of the RF MEMS switch. As discussed earlier using the low spring constant depicts the compromise of the material properties like Young's modulus. As the DMTL consist of mechanically actuated element which involves in higher amounts stress and strain that might effect the switch durability. So there exists a limit in minimising the spring constant. On other hand the spring constant is related to the resonant frequency of beam which governs the overall speed of the device. It can be explained the charge and discharge cycle of the device is considered to be a on switch action. The amount of time (ts) taken by the device to either mover push to pull (contact and Non-contact) is considered as the speed of the devices. In terms of resonant frequency of the beam it can expressed as

$$
t s=\frac{3.67 * V_{\mathrm{p}}}{V_{s \omega}}
$$

The voltage $(\mathrm{Vp})$ is considered to be the pull in voltage which is directly proportional and Source voltage (Vs) and frequency of the beam are inversely proportional. As in most of the DMTL devices, it was explained in literature that source voltage (Vs) should be 1.4 times of actuation voltage which can be replaced in the above equation.

$$
\mathrm{Ts}=\frac{2.6^{\circ}}{\omega}[8]
$$

From the above equation, it was evident that

$$
\text { ts }=2.67 \sqrt{m} / K
$$

So, for the device to faster, the switching time should be less, which implicates that mass should be less and the spring constant should be higher. So there is always a tradeoff between the pull-in voltage and the switching time in phase shifters so that once has to chose the design according to the applications. In order to minimise this tradeoff a technique, referred as the stress concentration regions are applied to DMTL, discussed in few recent articles $[8,10]$.

\section{B. Meshing}

The adaptive mesh technique is used where both mechanical and electric part are differentiated with triangular and rectangular mesh so that the solution converges to a finite value. As the beam is tend deflect a fine mesh was used to solve for the Eigen frequency analysis to most absolute value.

\section{Analysis of DMTL}

As the analysis is considered, it was carried as two different approaches, one approach is analysing the basic element of the DMTL phase shifter, which is nothing but the Reconfigurable RF MEMS switch and other part is other part is 5 bit DMTL phase shifter that can show phase difference in $11.25,22.5,45,90,180$ degree's where a series of array elements are considered for the design. The reconfigurability of the switch is approached with the double push-pull effect, by making the free boundary condition in pull stage and in push stage they are meant to fixed, this makes the centre diaphragm to move in push and pull stages which results in parasitic capacitance change. But this cents diaphragm is governs the switching speed of the device and this intern depends upon the rectangular cantilever supports that make the diaphragm to move. So as the rectangular cantilever are least responsive when compared with the triangular cantilever are replaced and frequency improvement of the beam is observed.

\section{Materials and Boundary conditions}


As materials are considered in crucial parameter in design of the Phase shifter as per tuning the devices and feasible fabrication flow. The approach is followed with the silicon/Roger FR4 as the substrate. Oxide layers as the dielectric layer. But in case of the beam, where spring constant and poisons ration governs both the pull-in voltage and switching speed, a parametric sweep was adopted between the Copper, Aluminum and Gold which suitable commercial and satellite applications[11-14]. In boundary conditions, that are applicable to be beam with respect to the structural physics and in the other hand electrostatic conditions are considered with the entire device. The reconfigurability of the switch is approached with the double push-pull effect, by making the free boundary condition in pull stage and in push stage they are meant to fixed, this makes the centre diaphragm to move in push and pull stages which results in parasitic capacitance change. It was observed in the analysis that gold having higher quality factor

\section{E. Discussion:}

The approach that was considered in this paper is completely based on the mechanically tuning of the DMTL, which is approached by designing a Reconfigurable RF MEMS switch. As it was discussed in the earlier sections that reconfigurable switch that can operate a different bandwidth's have wider application rather than going with the single bandwidth device. But these devices tend to face different resolution at each bandwidth that degrades the overall performance of the device. In general approach the reconfigurable design is achieved by loading with parasitic capacitance line or inductor line that can be used to tune the device to operate at different bandwidths. But in this approach a mechanical method was adopted to achieve that would avoid the leakage current losses. The reconfigurability of the switch is approached with the double push-pull effect, by making the free boundary condition in pull stage and in push stage they are meant to fixed, this makes the centre diaphragm to move in push and pull stages which results in parasitic capacitance change[12].

$$
\Delta \phi=\frac{\omega Z_{0} \sqrt{\varepsilon_{\omega}}}{c}\left(\frac{1}{Z_{1}}-\frac{1}{Z_{2}}\right) \mathrm{rad} / \mathrm{m}
$$

But as the complexity of design of switch is higher, the challenges like switching speed and pull voltage are observed in this design. As the electric approaches that supports to increase the switching speed and decrease the pull-in voltage are saturated[17-19], this paper as concentrated in adopting the mechanical approach where the rectangular cantilever are replaced with triangular cantilevers and in parallel a stress concentration region was applied. It was observed in that in the engine frequency analysis, there is considerable increase in the frequency of the centre diaphragm which showing around maximum of $17 \%$ increase than the rectangular structure and intact one mode the overall frequency was increased. Where as in case of single standalone switch there is average of $1.72 \mathrm{KHz}$ improvement. This would improve the overall switch speed by 5 to 10 micro seconds on average. So the DMTL acts as the improved version of the existing reconfigurable devices. The main intention was to prove that the mechanical tuning is one such area where the RF Switches and based DMTL phase shifter can be improved and this was not much concentrated in the literature part. So a comparison was made between the rectangular base and triangular base DMTL phase shifter and a 5 bit design approach was adopted in this paper. A material base parametric sweep is conducted with different materials of the beam that would be useful in analysing the devices for different applications. The overall capacitance between both the design was analysed where triangular cantilever has charge which means the charge and discharge cycles are faster than that of counter part and where maximum deflection of the diaphragm is observed in triangular design as well which gives better charge and discharge cycles by avoiding the parasitic and hysteresis capacitance.

TABLE I. DIMENSION OF RF MEMS SWITCH

\begin{tabular}{|c|c|c|c|}
\hline SL.No. & \multicolumn{3}{|c|}{ Dimensions of the Device } \\
\hline 1 & Length of the plate & Wpl & $170 \mathrm{um}$ \\
\hline 2 & Width of the plate & Wow & $170 \mathrm{um}$ \\
\hline 3 & Length of rectangular canticlever & $\mathrm{Rl}$ & 60um \\
\hline 4 & Width of rectangular cantilever & & 20um \\
\hline 5 & Center plate dimensions & & $40 * 40 u m$ \\
\hline 6 & Anchor length & & 220um \\
\hline 7 & Anchor width & & 20um \\
\hline 8 & Total device thickness & & $2 \mathrm{um}$ \\
\hline \multirow[b]{2}{*}{ SL.No. } & \multicolumn{3}{|c|}{ Resonant frequency modes for beam } \\
\hline & Resonant modes & Triangular & $\begin{array}{l}\text { Rectang } \\
\text { ular }\end{array}$ \\
\hline 1 & Mode 1 & 41473 & 41295 \\
\hline 2 & Mode 2 & 57884 & 56914 \\
\hline 3 & Mode 3 & 62250 & 62148 \\
\hline 4 & Mode 4 & 829656 & 82816 \\
\hline
\end{tabular}



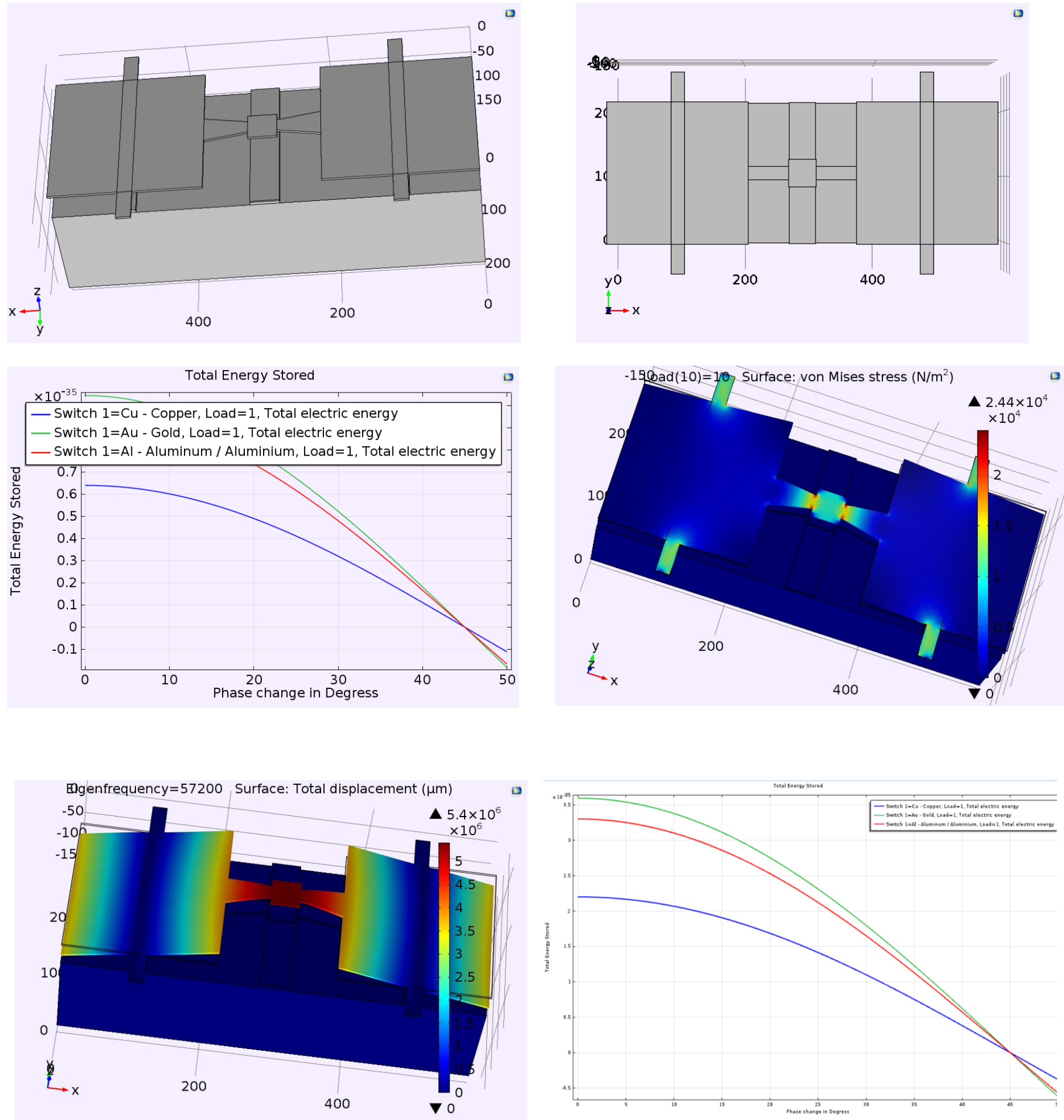

Fig 2 a) Push pull configuration triangular RF MEMS switch B) Rectangular switch configuration c) energy stored in traingular switch . D) stress developed in traingular switch during actuation E) Total displacment F) Energy stored in rectangular switch/

Published By:

Blue Eyes Intelligence Engineering

\& Sciences Publication

(C) Copyright: All rights reserved. 

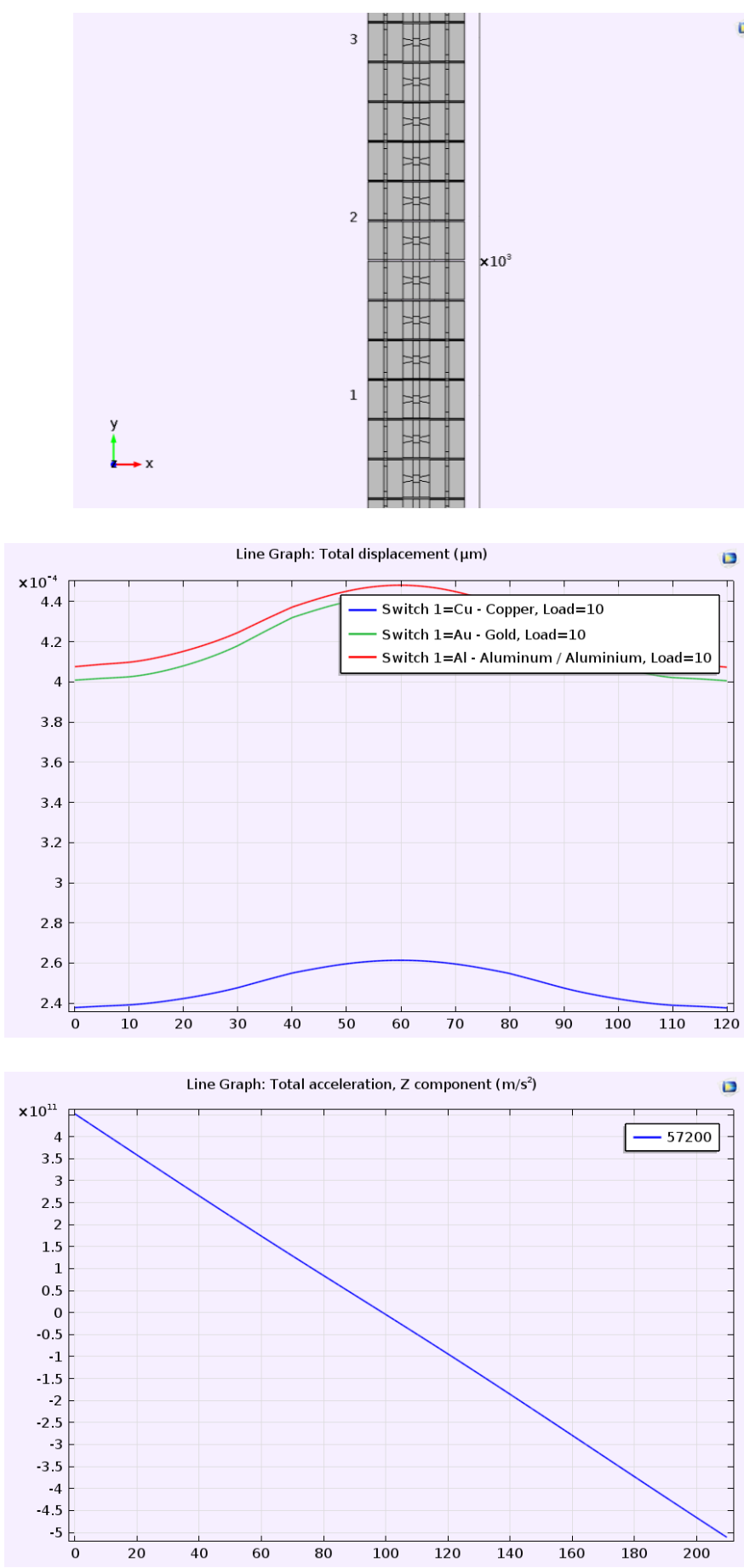

0
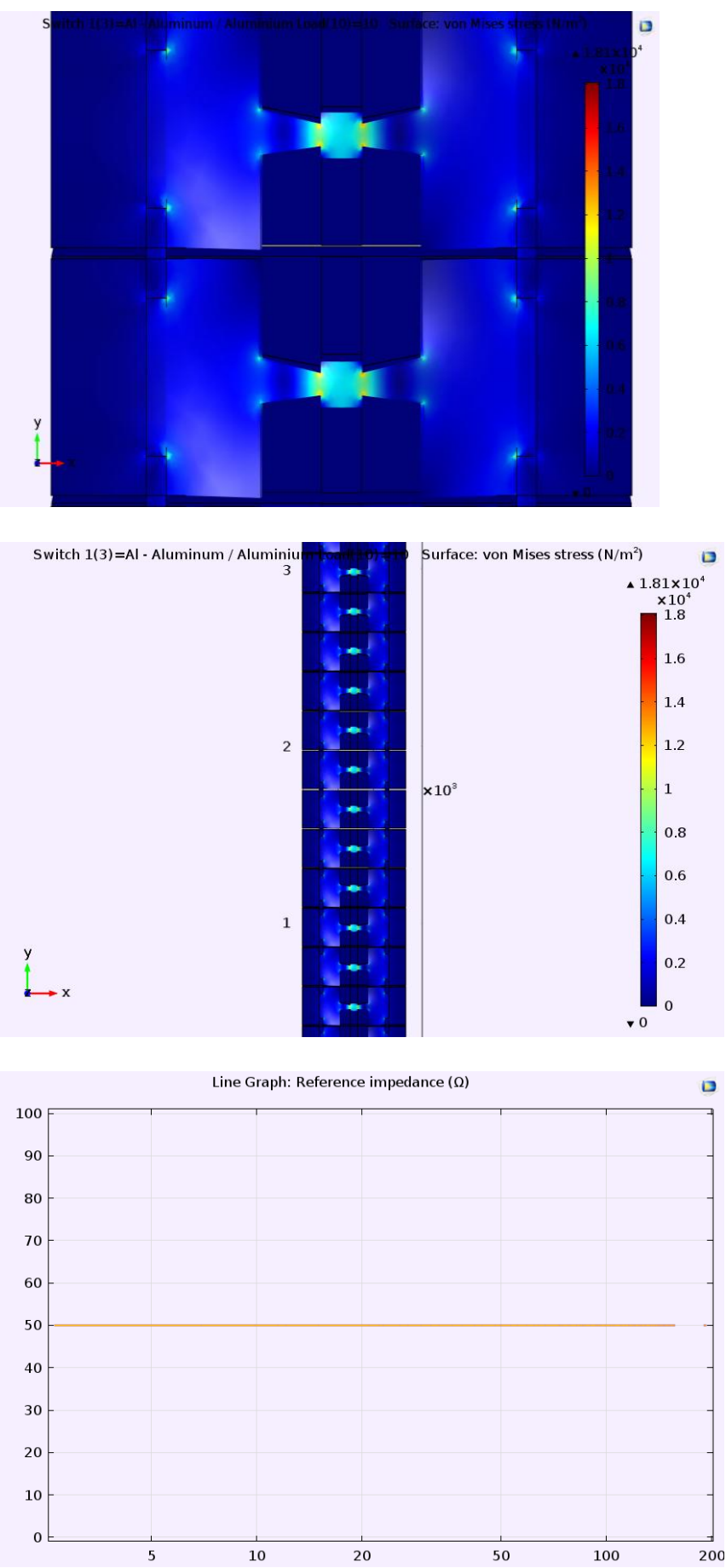

Fig 3. A) Triangular DMTL with 5 bit operation b) stress analysis under actuation mode c)total displacement of the beam with parametric sweep d)actuation of 180degrees $n$ stress developed e0acceleration of the beam $f$ )characteristic impedance of the device.

\section{Conclusion}

The switching speed and the resonant frequency of the RF devices plays a key role in analysing the device speed in most of the applications and they mainly rely on the principle components of the RF device phenomenon. This work provokes about the RF MEMS switch which is integrated with the DML shifter. The shifter tend to have triangular mechanical structure with a stress factor which in turn designed to enhance the speed and the efficiency of the DMTL phase shifter along with RF switch stability. As DMTL shifter is correlated with RF series switch, improving
Published By:

Blue Eyes Intelligence Engineering \& Sciences Publication

the switching speed will result in improved response time for the overall design. For the actuation phenomenon and device tuning, the cantilever with rectangular configuration was replaced with the triangular architecture, where a switching speed is improved about $17 \%$ is being achieved shown table 1. This would develop and enable better application in terms of reconfigurable phase shifter which can be operated at the wider bandgap applications.

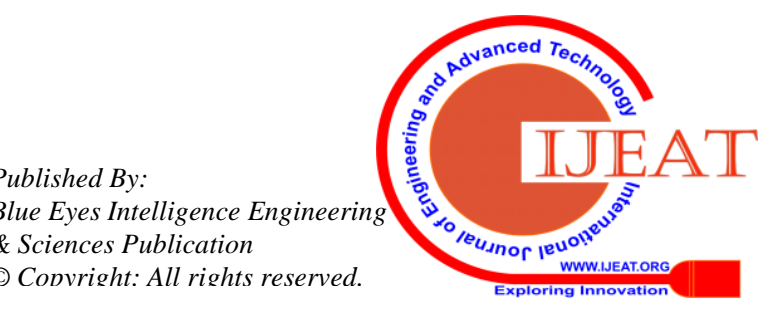


These device needs further improvisation in terms of adopting the stress region, enhancing this parameter might help the future device with better device performance scale

\section{REFERENCES}

1. Petersen KE 1982 Silicon as a mechanical material Proc. IEEE 70 420-57.

2. Srihari, G., and T. Shanmuganantham. "Design and Analysis of MEMS Based RF Capacitive Switch for DMTL Phase Shifter." 2019 TEQIP II Sponsored International Conference on Microwave Integrated Circuits, Photonics and Wireless Networks (IMICPW). IEEE, 2019.

3. ChauKH-L,LewisSR,ZhaoY,HoweRT,BartSFand Marcheselli R G 1995 An integrated force-balanced capacitive accelerometer for low-g applications Tech. Digest, 8th Int. Conf. on Solid-State Sensors and Actuators pp 593-6.

4. Koul, Shiban Kishen, and Sukomal Dey. Radio Frequency Micromachined Switches, Switching Networks, and Phase Shifters. CRC Press, 2019.

5. Burns DW, Horning RD, Herb WR, Zook JDand Guckel H 1995 Resonant microbeam accelerometers Tech. Digest, 8th Int. Conf. on Solid-State Sensors and Actuators pp 659-62

6. Huang, Qinwen, et al. "A potential degradation mechanism in RF MEMS ohmic switches." International Journal of Modern Physics B 33.14 (2019): 1950135.

7. Chavan A V and Wise K D 1997 A batch-processed vacuum-sealed capacitive pressure sensor Tech. Digest, 1997 Int. Conf. on Solid-State Sensors and Actuators pp 1449-52

8. Rao, K. Srinivasa, et al. "Design, Modeling and Analysis of Perforated RF MEMS Capacitive Shunt Switch." IEEE Access 7 (2019): 74869-74878

9. Lee S-S, Motamedi E and Wu M C 1997 Surface-micromachined free-space fiber optic switches with integrated microactuators for optical fiber communication systems Tech. Digest, 1997 Int. Conf. on Solid-State Sensors and Actuators pp 85-8.

10. Aditya, A. L. G. N., and Elizabeth Rufus. "MEMS Capacitive Switch with Stress Concentration Regions for Wide band Applications Applications-Switching time Improvement for High Speed Applications." 2019 International Conference on Vision Towards Emerging Trends in Communication and Networking (ViTECoN). IEEE, 2019.

11. Y. Huang, J. Bao, X. Li, Y. Wang, and Y. Du, "A 4-bit switched-line phase shifter based on MEMS switches," 2015 IEEE 10th Int. Conf. Nano/Micro Eng. Mol. Syst. NEMS 2015, pp. 405-408, 2015

12. J. S. Hayden and G. M. Rebeiz, "Very low-loss distributed X-band and Ka-band MEMS phase shifters using metal-air-metal capacitors," IEEE Trans. Microw. Theory Tech., vol. 51, no. 1 II, pp. 309-314, 2003.

13. Nguyen, C.T.-C.; Katehi, L.P.B.; Rebeiz, G.M. Micromachined Devices for wireless communications. Proc. IEEE 1998, 86, 1756-1768.

14. Yao, J.J. RF MEMS from a device perspective. J. Micromech. Microeng. 2000, 10, R9-R38.

15. Tilmans, H.A.; Raedt, W.D.; Beyne, E. MEMS for wireless communications: 'From RF-MEMS components to RF-MEMS-SiP’. J. Micromech. Microeng. 2003, 13, S139-S163.

16. R. D. E. Ensayo, R. Sergio, and E. Trejos, "Análisis químico foliar," vol. 01, p. 6558, 2000.

17. L. Voltage and F. R. R. Switches, "Laterally Movable Triple Electrodes Actuator toward Low Voltage and Fast Response RF-MEMS Switches," 2019.

18. N. Haridas, "Design and Simulation of a 3-Bit DMTL Phase Shifter for Wideband Applications," pp. 2-6.

19. H. Search, C. Journals, A. Contact, M. Iopscience, and I. P. Address, "Design , development and characterization of an x-band 5 bit DMTL phase shifter using an inline MEMS bridge and MAM capacitors," vol. 095007.

20. Dey, Sukomal, and Shiban K. Koul. "10-35-ghz frequency reconfigurable rf mems 5-bit dmtl phase shifter uses push-pull actuation based toggle mechanism." 2014 IEEE International Microwave and RF Conference (IMaRC). IEEE, 2014

\section{AUTHORS PROFILE}

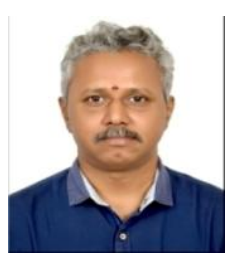

T. Shanmuganantham, received B.E. degree in Electronics and Communication Engineering from University of Madras, M.E. degree in Communication Systems from Madurai Kamaraj University and Ph.D. (Gold Medal) in the area of Antennas from National Institute of Technology, Tiruchirappalli, India under the guidance of Dr. S. Raghavan, Professor/ECE, NITT. He has 23years of teaching experience in various reputed Engineering colleges such as PKIET, SSN College of Engineering, National Institute of Technology and Science. He is currently working as Assistant Professor in the Department of Electronics Engineering, School of Engineering \& Technology, Pondicherry Central University, Pondicherry. His research interest includes Antennas, Microwave/Millimeter-Wave Circuits and Devices, MEMS/NEMS. He has published 375 research papers in various National and International level Journals and Conferences. His biography was included in Marquis who is who in the world, USA in the year 2010.He received Fellow in ATMS and a member in IEEE, Life Member in ISSS, IETE, IE (India), CSI (India), Society of EMC, OSI, ILA, ISI and ISTE.

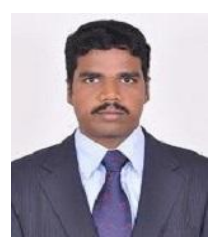

MEMS/NEMS.
G Srihari, received B.Tech. degree in Electronics and Communication Engineering from JNTU Hyderabad, M.Tech. degree in VLSI System Design from JNTU Hyderabad and Currently as research scholar Department of Electronics Engineering, School of Engineering \& Technology, Pondicherry Central University, Pondicherry. His research interest includes 